\title{
Estudo da micobiota em conjuntiva sadia de diabéticos, residentes na área urbana da cidade de São Paulo - Brasil
}

\author{
Study of mycobiota in the healthy conjunctiva of diabetics who reside in the \\ urban area of the city of São Paulo, Brazil
}

\author{
Alfredo José Muniz de Andrade ${ }^{1}$ \\ Ana Luisa Höfling'-Lima ${ }^{2}$ \\ Maria Cecília Zorat Yu ${ }^{3}$ \\ Patricio Godoy $^{4}$ \\ Olga Fischman Gompertz ${ }^{5}$ \\ Sabrina de Souza Bonfim ${ }^{6}$ \\ Francisco Eudes Muniz de Andrade ${ }^{7}$
}

Trabalho realizado no Departamento de Oftalmologia da Universidade Federal de São Paulo - UNIFESP - São Paulo (SP) - Brasil.

Parte da Tese apresentada à UNIFESP para obtenção do Título de Doutor em Medicina.

${ }^{1}$ Doutor em Medicina pela Universidade Federal de São Paulo - UNIFESP - São Paulo (SP) - Brasil.

${ }^{2}$ Professora Titular do Departamento de Oftalmologia da UNIFESP - São Paulo (SP) - Brasil.

3 Biomédica responsável pelo laboratório de Doenças Externas Oculares do Departamento de Oftalmologia da UNIFESP - São Paulo (SP) - Brasil.

${ }^{4}$ Doutor em Ciências pela UNIFESP - São Paulo (SP). Disciplina de Doenças Infecciosas e Parasitárias, LEMI UNIFESP - São Paulo (SP) - Brasil.

${ }_{5}^{5}$ Professora Adjunto-Doutora do Departamento de Microbiologia, Imunologia e Parasitologia da UNIFESP - São Paulo (SP) - Brasil.

${ }^{6}$ Tecnóloga Oftálmica do Departamento de Oftalmologia da UNIFESP - São Paulo (SP) - Brasil.

Acadêmico do Curso Médico da Universidade Federal de Pernambuco - UFPE - Recife (PE) - Brasil.

Endereço para correspondência: Alfredo José Muniz de Andrade. Rua Ibiapaba, 89/402 - Recife (PE)

CEP 52051-100

E-mail: alfredo@andrade.med.br

Recebido para publicação em 28.05.2004

Versão revisada recebida em 16.03.2005

Aprovação em 15.08.2005

\section{RESUMO}

Objetivos: Determinar a micobiota de conjuntiva sadia em indivíduos diabéticos, segundo tipo de diabetes, idade, sexo, tempo de doença, tipo de tratamento e estádio da retinopatia. Estabelecer a micobiota anemófila nas salas de colheita. Métodos: Estudo transversal de 803 diabéticos residentes na zona urbana de São Paulo - SP/Brasil. Foi usado para primoisolamento o meio de cultivo ágar Sabouraud dextrose com cloranfenicol e para identificação dos fungos filamentosos a chave de De Hoog. Resultados: Dos diabéticos avaliados, 6,6\% (53/803) apresentavam diabetes tipo 1 e $93,4 \%$ (750/803) tipo 2 . Os cultivos positivos para fungos em conjuntiva de diabéticos foi 4,2\% (34/803), sendo $1,9 \%(1 / 53)$ nos diabéticos tipo 1 e 4,4\% (33/740) nos diabetes tipo 2 ( $p=0,720)$. Não foi verificada associação estatisticamente significante quanto à presença ou não de isolamentos de fungos em relação idade $(\mathrm{p}=0,575)$, sexo $(\mathrm{p}=0,517)$, tempo de doença $(\mathrm{p}=0,633)$, tipo de tratamento $(\mathrm{p}=0,422)$ e estádio de retinopatia diabética $(\mathrm{p}=0,655)$ desses indivíduos. Todos os fungos identificados foram filamentosos: Aspergillus spp. representou 59,5\% (25/42) dos isolamentos sendo 47,6\% (20/42) Aspergillus niger. Ocorreu crescimento de fungos anemófilos do ar ambiente da sala, observando-se coincidências entre as espécies isoladas no ar e na conjuntiva. Conclusões: Foi identificada presença de micobiota em conjuntiva sadia de diabéticos, não havendo associação entre a maior positividade de isolamentos fúngicos e o tipo de diabetes, idade, sexo, tempo de doença, tipo de tratamento e estádio da retinopatia diabética. Nas salas de colheita foi identificada micobiota anemófila.

Descritores: Conjuntiva/microbiologia; Fungos/isolamento \& purificação; Diabetes mellitus: Retinopatia diabética; Contagem de colônia microbiana; Aspergillus niger/isolation \& purification; Aspergillus/isolation \& purification; População urbana

\section{INTRODUÇÃO}

A micobiota ocular normal engloba os fungos presentes nas pálpebras, canais lacrimais e superfície ocular ${ }^{(1)}$. Para grande maioria de autores os fungos isolados em conjuntivas sadias são originados da biota anemófila, pois não foram detectadas evidências de que fungos pertençam à biota permanente em olhos sadios, devido a não repetição dos resultados obtidos em cultivos seriados de conjuntiva ${ }^{(2-16)}$. Existe descrições que as mudanças verificadas no número e tipo das espécies integrantes da micobiota em conjuntiva, variam segundo a estação do ano ${ }^{(11-12,17)}$ e uso tópico de 
corticosteróide isolado ou associado a antibiótico ${ }^{(3-4,11,18-19)}$. A dispersão de fungos no ar pode ser influenciada por fatores, como: precipitação, umidade relativa do ar, velocidade do vento, temperatura do ar e altitude ${ }^{(13)}$.

Estudos realizados em vários países, a partir de 1935 que investigaram a micobiota em conjuntivas sadias, apresentam resultados que variaram de zero a $84,8 \%$ de cultivos positivos. As frequiências de isolamentos de fungos em conjuntiva sadia, nem sempre são passíveis de comparação. Pois, os autores expressaram essa frequiência de diversas formas, ou seja, às vezes segundo número de indivíduos e em outras ocasiões, segundo o número de olhos com cultivos positivos ${ }^{(2-6,8-12,14-15,18-32)}$.

$\mathrm{O}$ diabetes melito (DM) constitui um grupo de doenças caracterizadas por hiperglicemia crônica, resultante de alterações na produção ou ação da insulina e outros distúrbios no metabolismo de carboidratos, lipídeos e proteínas. A hiperglicemia crônica é associada a lesões progressivas nos olhos, rins, nervos, vasos sangüíneos e coração ${ }^{(33)}$.

Os indivíduos diabéticos com glicemias elevadas são naturalmente expostos às infecções superficiais ou sistêmicas por fungos oportunistas ${ }^{(34)}$.

O conhecimento dos componentes da micobiota conjuntival orienta formulações das hipóteses diagnósticas nas infecções oculares. Para se caracterizar as infecções micóticas oculares, é importante a certeza da existência, ou não, de fungos, como integrante da biota transitória ou permanente na conjuntiva $^{(16)}$

$\mathrm{Na}$ literatura mundial pesquisada, não foram encontrados estudos sobre a micobiota em conjuntiva sadia de indivíduos diabéticos. O presente estudo teve como objetivos realizar raspados em conjuntiva sadia de indivíduos diabéticos residentes em zona urbana da cidade de São Paulo, para determinar a micobiota e verificar associações da ocorrência ou não de isolamentos fúngicos nesses indivíduos segundo tipo de diabetes, idade, sexo, tempo de doença, tipo de tratamento e estádio da retinopatia diabética e estabelecer a micobiota anemófila nas salas de colheita dos raspados de conjuntiva.

\section{MÉTODOS}

Foi realizado estudo transversal em amostra não probabilística, utilizando raspados de conjuntiva sadia de indivíduos diabéticos de ambos os sexos e idade variando entre $18 \mathrm{e}$ 90 anos, com diagnóstico de diabetes comprovado pela história clínica e uso de medicamentos específicos. Os pacientes foram atendidos no Mutirão Olho Diabético na Universidade Federal de São Paulo, no período de junho de 2001 a março de 2002 , situado latitude sul $23^{\circ} 35^{\prime} 58^{\prime \prime}$, longitude oeste $46^{\circ} 38^{\prime} 23^{\prime \prime}$ de, altitude $779 \mathrm{~m}$. Os critérios de exclusão foram presença de doença ocular, uso de medicação tópica ou lentes de contato nos últimos 30 dias, ser portador de doença que possa modificar a resposta imune (exemplo: leucemia, linfoma, neoplasias, infecções virais e desnutrição) e procedência da zona rural.

O protocolo desse estudo foi aprovado pelo Comitê de
Ética da Universidade Federal de São Paulo pelo processo n⿳ 464/01, em 29/06/2001 e obedeceu aos princípios da declaração de Helsique. Após obtenção do consentimento livre permitido dos participantes, os indivíduos foram entrevistados e então procedido à colheita das amostras de conjuntiva. $\mathrm{O}$ horário de colheita foi das $8 \mathrm{~h} 00$ às $15 \mathrm{~h} 00$ e a média da temperatura do ar variou de 17 a $24^{\circ} \mathrm{C}$. A intensidade de precipitação mensal variou de 29 a $305 \mathrm{~mm}$.

\section{Pacientes}

Os 803 diabéticos foram caracterizados segundo idade, sexo, tipo de DM, valor da última glicemia plasmática em jejum (GPJ), tempo de doença, tipo de tratamento para DM e avaliação da retina utilizando oftalmoscopia binocular indireta. Conforme os resultados dos mapeamentos de retina, os diabéticos foram classificados em quatro grupos: 1) normal, 2) retinopatia não proliferativa (RNP) sem maculopatia, representando a junção dos indivíduos com estádio leve, moderado e grave, 3) RNP com maculopatia e 4) retinopatia proliferativa (RP) ${ }^{(35)}$. Nos casos com ocorrência de retinopatia diabética assimétrica entre os olhos, foi considerado o olho com estádio mais avançado.

\section{Técnicas microbiológicas}

Foram colhidas as amostras de conjuntiva dos pacientes uma vez a cada mês.

$\mathrm{O}$ teste de esterilidade, por amostragem, nos lotes das zaragatoas alginatadas esterilizadas (BIONETE HA ${ }^{\circledR}$, Biolog, São Paulo, Brasil) foi realizado três dias antes de cada colheita.

Os raspados de conjuntiva foram colhidos em uma sala, adotando determinados cuidados: limpeza do chão e paredes com água e sabão, janelas fechadas, aparelhos condicionadores de ar desligados e presença somente de dois pesquisadores por vez na sala, usando luvas e máscara faciais. Somente um pesquisador efetuou as colheitas.

Os participantes tiveram ambos os olhos pesquisados, sem anestesia. As manipulações dos materiais e as colheitas foram realizadas próximo à chama de lamparina. A zaragatoa foi umedecida em solução salina esterilizada (Solução de Cloreto de Sódio 0,9\% ${ }^{\circledR}$, Aster, Sorocaba, Brasil) e aplicada com movimento de rotação e em deslizamento sobre a superfície da conjuntiva tarsal e fundo de saco inferior, no sentido naso-temporal e temporo-nasal, sem tocar em margens palpebrais ou cílios. Repetiu-se a mesma técnica de colheita para o outro olho. A semeadura foi realizada no meio de Sabouraud dextrose ágar (Ágar Sabouraud Dextrose ${ }^{\circledR}$, Oxoid, Hants, Inglaterra) acrescido de cloranfenicol (Cloranfenicol ${ }^{\circledR}$, Ducto, São Paulo, Brasil) $200 \mathrm{mg} \cdot \mathrm{l}^{-1}$, executando trajetória em curva senóide e acompanhada de movimentos de rotação do eixo da zaragatoa. Foi usado uma placa de Petri de $80 \mathrm{~mm}$ (Placas de Petri descartável ${ }^{\circledR}$, J. Prolab, Curitiba, Brasil) para cada paciente, sendo que cada olho teve sua estria de semeadura identificada, em espaço imediatamente abaixo no meio de cultivo. As placas foram armazenadas em ambiente fechado e umedecido.

A pesquisa de fungos anemófilos na sala de colheita ocorreu simultaneamente com as da conjuntiva, utilizando o méto- 
do da gravidade. Consistindo em colocar uma placa com meio de cultivo Sabouraud dextrose ágar acrescido de cloranfenicol $200 \mathrm{mg} . \mathrm{l}^{-1}$, aberta ao ambiente por 30 minutos.

A leitura das placas foi diária, iniciando-se 48 horas após a colheita, até completar 30 dias. Cultivos com crescimentos em estria foram encaminhados para identificação imediata. $\mathrm{O}$ desenvolvimento de fungos e bactérias, fora dos locais de semeadura, foi considerado como contaminação, sendo desprezados.

As placas com amostras dos fungos anemófilos obedeceram à mesma sequiência de procedimento dos meios com amostras de conjuntiva.

Para a identificação dos fungos, realizaram-se exames macroscópico e microscópico das colônias suspeitas, utilizando lactofenol-azul de algodão. As normatizações de De Hoog et al., foram seguidas para fungos filamentosos, baseadas nas suas características morfológicas ${ }^{(36)}$. Os cultivos eram mantidos à temperatura ambiente. Sempre que necessário, foi feito microcultivo nos meios de cultivo ágar batata dextrose, ágar extrato de malte ou ágar aveia para melhor observação e caracterização do microrganismo.

\section{Análise estatística}

Para determinar o tamanho da amostra desse estudo, utilizou-se como prevalência estimada de indivíduos com cultivos positivos para fungos em conjuntiva sadia, os resultados observados em estudos, que tiveram mais de 100 pessoas avaliadas para este tipo de pesquisa, sendo determinada a prevalência 11,0\% (284/2579) de indivíduos com isolamentos positivos $^{(2,6,8,10,24-25,28-29)}$, margem de erro $5,0 \%$ e confiabilidade $95,0 \%$. Considerando erro de $2,5 \%$, o tamanho da amostra calculado foi 602 pessoas, sendo no presente estudo realizado com 803 indivíduos.
Para análise dos dados foram usados: teste Qui-Quadrado $\left(\chi^{2}\right)$ de independência, teste Exato de Fisher (quando as condições para utilização do $\chi^{2}$ não foi verificada), teste de MannWhitney e o teste de comparação de duas proporções. Sendo também determinado Odds Ratio (OR) e Intervalo de Confiança (IC) de 95,0\% para este parâmetro, considerando-se a última categoria como referência. Para se verificar o número de concordâncias entre os resultados dos mapeamentos de retina dos diabéticos, foi obtido o escore de Kappa ${ }^{(37)}$. O nível de significância, considerado nas decisões dos testes estatísticos foi $5,0 \%$, sendo usado programa de computador para a obtenção desses cálculos (SAS ${ }^{\circledR}$ Version 6.12, SAS Institute Inc., Cary-NC, EUA).

\section{RESULTADOS}

A média de idade dos diabéticos foi 57,44 anos (dp 11,94 anos, idade mínima-máxima 18-89 anos, coeficiente de variação de 20,79\%), sendo 6,6\% (53/803) portadores de DM tipo 1 e 93,4\% (750/803) do tipo 2. A média de idade dos indivíduos com DM tipo 1 foi 40,17 anos (dp 13,72 anos, idade mínimamáxima 18-69 anos, coeficiente de variação de $34,15 \%$ ) e nos diabéticos tipo 2 foi 58,66 anos (dp 10,82 anos, idade mínimamáxima 18-89 anos, coeficiente de variação de $18,45 \%$ ). A idade dos indivíduos com DM tipo 2 foi mais elevada que a dos diabéticos tipo 1 , sendo verificada diferença significante $(\mathrm{p}<0,001)$.

A distribuição dos indivíduos diabéticos tipo 1 e tipo 2 segundo sexo, glicemia plasmática em jejum (GPJ), tempo de doença e estádio da retinopatia diabética são discriminados na tabela 1. Não se comprova associação significante do tipo

\begin{tabular}{|c|c|c|c|c|c|c|}
\hline \multirow{3}{*}{ Variáveis } & \multicolumn{4}{|c|}{ Diabéticos $(\mathrm{N}=803)$} & \multirow{3}{*}{$\mathbf{p}^{\dagger}$} & \multirow{3}{*}{ OR (IC 95,0\%) } \\
\hline & \multicolumn{2}{|c|}{ Tipo 1} & \multicolumn{2}{|c|}{ Tipo 2} & & \\
\hline & $\mathbf{N}$ & $\%$ & $\mathbf{N}$ & $\%$ & & \\
\hline \multicolumn{7}{|l|}{ Sexo } \\
\hline Masculino & 20 & 37,7 & 322 & 42,9 & 0,460 & $0,81 \quad(0,70-2,20)$ \\
\hline Feminino & 33 & 62,3 & 428 & 57,1 & & 1,00 \\
\hline \multicolumn{7}{|l|}{ GPJ (mg.dl-1) } \\
\hline Boa & 25 & 47,2 & 367 & 48,9 & 0,970 & $0,93(0,52-1,69)$ \\
\hline Aceitável & 6 & 11,3 & 82 & 10,9 & & $1,00 \quad(0,39-2,55)$ \\
\hline Ruim & 22 & 41,5 & 301 & 40,1 & & 1,00 \\
\hline \multicolumn{7}{|l|}{ Tempo doença (anos) } \\
\hline$\leq 5$ & 10 & 18,9 & 308 & 41,1 & $<0,001$ & $0,23(0,11-0,48)$ \\
\hline 6 a 10 & 8 & 15,1 & 190 & 25,3 & & $0,30 \quad(0,14-0,67)$ \\
\hline$\geq 11$ & 35 & 66,0 & 252 & 33,6 & & 1,00 \\
\hline \multicolumn{7}{|l|}{ Estádio RD } \\
\hline Normal & 25 & 6,2 & 379 & 93,8 & 0,587 & $0,51 \quad(0,26-1,01)$ \\
\hline RNP s/maculopatia & 10 & 5,3 & 177 & 94,7 & & $0,44(0,19-1,01)$ \\
\hline RNP c/maculopatia & 3 & 3,7 & 77 & 96,3 & & $0,30(0,08-1,08)$ \\
\hline $\mathrm{RP}$ & 15 & 11,4 & 117 & 88,6 & & 1,00 \\
\hline
\end{tabular}


de DM com sexo ou glicemia plasmática em jejum desses indivíduos no momento do estudo. A porcentagem de indivíduos com mais de 11 anos de evolução da doença foi maior nos diabéticos tipo 1 , sendo também observada maior freqüência de diabéticos tipo 2 com menos de cinco anos e com seis a dez anos de doença; verificando-se diferença significante entre os dois grupos, conforme valor de $\mathrm{P}$ e intervalos para OR que excluem a unidade. Não se evidencia diferença significante entre o grau de retinopatia diabética presente nos diabéticos tipo 1 e tipo 2 .

Das avaliações de retina dos diabéticos em relação ao exame do olho direito e do esquerdo, foram observadas que $83,3 \%(669 / 803)$ apresentaram exames de retina simétricos e $16,7 \%$ (134/803) com manifestação assimétrica. O escore de Kappa foi 0,73 , evidenciando que as alterações na retina pelo DM, em ambos os olhos, não eram independentes $(\mathrm{p}<0,001)$.

Dos diabéticos avaliados, 47,4\% (383/803) faziam tratamento com hipoglicemiante oral, $36,6 \%$ (294/803) usavam insulina e $15,7 \%$ (126/803) seguiam somente dieta específica para DM.

Nos testes de esterilidade, aplicados às zaragatoas alginatadas e meios de cultivo utilizados no estudo, não foi observado crescimento de microrganismos.

Na tabela 2, observa-se a distribuição dos indivíduos diabéticos, segundo cultivo positivo, por ordem de identificação, olho acometido, espécie fúngica e número de colônias isoladas. O cultivo positivo para fungo ocorreu em 4,2\% (34/803) dos indivíduos diabéticos. Dos 34 indivíduos cujos olhos tiveram isolamentos positivos para fungo, 30 indivíduos (30 olhos) apresentaram comprometimento monocular e 4 (8 olhos) binocular. Dos isolamentos observados nos diabéti$\cos , 71,4 \%$ (30/42) ocorreram em exames realizados em olho direito e $28,6 \%$ (12/42) em olho esquerdo. A identificação de uma espécie fúngica, por olho, ocorreu em 40 olhos e duas espécies fúngicas diferentes por olho, foram identificadas em quatro olhos. A classificação morfológica das espécies fúngicas isoladas, revelou $83,3 \%$ (35/42) de fungos filamentosos hialinos, 14,3\% (6/42) de filamentosos demácios e 2,4\% (1/42) não apresentava estrutura de reprodução, sendo classificado como Mycelia sterilia. Não houve isolamento de fungos leveduriformes na conjuntiva dos indivíduos estudados. Verificou-se também, que 59,5\% (25/42) dos isolamentos foram do gênero Aspergillus e 47,6\% (20/42) das espécies foram Aspergillus niger. O número de colônias foi baixo nos 42 olhos com cultivo positivo, variou de uma a onze colônias (média 2,7 de colônias por indivíduos).

Na tabela 3 verifica-se a distribuição dos diabéticos tipo 1 e tipo 2, segundo a ocorrência ou não de isolamentos positivos para fungos, por idade, sexo, GPJ, tempo de doença, tipo de tratamento e estádio da retinopatia diabética. A análise estatística desses dados não demonstrou associação significante para nenhuma dessas variáveis, conforme valores de $\mathrm{p}$ e dos intervalos para OR.

Nos meses de julho, agosto e setembro de 2001 verificouse média de precipitação de chuvas de $59,3 \mathrm{~mm} / \mathrm{mês}$, com valores mínimo e máximo de 29 e $88 \mathrm{~mm} / \mathrm{mês}$, respectivamente.
Nos meses de outubro e novembro de 2001 e fevereiro e março de 2002, teve-se média de precipitação de chuvas de $250 \mathrm{~mm} /$ mês e valores mínimo e máximo de 200 e $305 \mathrm{~mm} / \mathrm{mês}$, respectivamente ${ }^{(38)}$

Na tabela 4 observa-se diferença significante entre as proporções de isolamentos fúngicos positivos e a média de precipitação de chuvas, ocorrendo $0,3 \%$ (1/349) dos isolamentos

\begin{tabular}{|c|c|c|c|}
\hline $\begin{array}{l}\text { Ordem } \\
\text { ident. }\end{array}$ & $\begin{array}{l}\text { Lateralidade } \\
\text { do olho }\end{array}$ & $\begin{array}{l}\text { Espécie } \\
\text { fúngica }\end{array}$ & $\begin{array}{l}\text { Número de } \\
\text { colônias* }\end{array}$ \\
\hline $1^{0}$ & $\mathrm{D}$ & Aspergillus fumigatus & 6 \\
\hline $2^{0}$ & $E$ & Phoma spp. & 1 \\
\hline $3^{\circ}$ & $E$ & Acremonium spp. & 1 \\
\hline $4^{\circ}$ & D & Acremonium spp. & 1 \\
\hline $5^{0}$ & $E$ & Paecilomyces variotti & 1 \\
\hline $6^{\circ}$ & $\mathrm{D}^{\dagger}$ & Aspergillus fumigatus & 4 \\
\hline $7^{\circ}$ & $\mathrm{D}^{\dagger}$ & Colletotrichum sp. & 4 \\
\hline $8^{\circ}$ & $\mathrm{D}^{\dagger}$ & Mycelia sterilia & 4 \\
\hline $9^{\circ}$ & $\mathrm{D}^{\dagger}$ & Phoma spp. & 4 \\
\hline $10^{\circ}$ & $\mathrm{D}^{\dagger}$ & Cladosporium cladosporoides & 5 \\
\hline $11^{\circ}$ & $D^{\dagger}$ & Ulocladium sp. & 5 \\
\hline $12^{0}$ & $D^{\ddagger}$ & Scytalidium lignicola & 1 \\
\hline $13^{\circ}$ & $E^{\ddagger}$ & Scytalidium lignicola & 2 \\
\hline $14^{\circ}$ & $\mathrm{D}^{\dagger}$ & Aspergillus niger & 4 \\
\hline $15^{\circ}$ & $\mathrm{D}^{\dagger}$ & Penicillium spp. & 4 \\
\hline $16^{0}$ & $\mathrm{D}$ & Penicillium citrinum & 1 \\
\hline $17^{\circ}$ & $E$ & Penicillium citrinum & 1 \\
\hline $18^{\circ}$ & $E$ & Paecilomyces lilacinus & 4 \\
\hline $19^{\circ}$ & D & Fonsecaea pedrosoi & 1 \\
\hline $20^{\circ}$ & D & Aspergillus spp. & 1 \\
\hline $21^{\circ}$ & $E$ & Aspergillus spp. & 5 \\
\hline $22^{\circ}$ & D & Penicillium citrinum & 1 \\
\hline $23^{\circ}$ & D & Aspergillus niger & 1 \\
\hline $24^{\circ}$ & D & Aspergillus niger & 1 \\
\hline $25^{\circ}$ & D & Aspergillus niger & 1 \\
\hline $26^{\circ}$ & $D^{\ddagger}$ & Aspergillus niger & 1 \\
\hline $27^{\circ}$ & $E^{\ddagger}$ & Aspergillus niger & 2 \\
\hline $28^{\circ}$ & D & Aspergillus niger & 5 \\
\hline $29^{\circ}$ & D & Aspergillus niger & 5 \\
\hline $30^{\circ}$ & D & Aspergillus niger & 2 \\
\hline $31^{\circ}$ & D & Aspergillus niger & 1 \\
\hline $32^{\circ}$ & D & Aspergillus niger & 11 \\
\hline $33^{\circ}$ & D & Aspergillus fumigatus & 1 \\
\hline $34^{\circ}$ & D & Aspergillus niger & 1 \\
\hline $35^{\circ}$ & $D^{\ddagger}$ & Aspergillus niger & 5 \\
\hline $36^{\circ}$ & $E^{\ddagger}$ & Aspergillus niger & 2 \\
\hline $37^{\circ}$ & $D^{\ddagger}$ & Aspergillus niger & 4 \\
\hline $38^{\circ}$ & $E^{\ddagger}$ & Aspergillus niger & 3 \\
\hline $39^{\circ}$ & $E$ & Aspergillus niger & 1 \\
\hline $40^{\circ}$ & E & Aspergillus niger & 1 \\
\hline $41^{\circ}$ & D & Aspergillus niger & 1 \\
\hline $42^{\circ}$ & D & Aspergillus niger & 3 \\
\hline
\end{tabular}


nos meses com precipitação de 59,3 mm/mês e 9,0\% (41/456) naqueles com $250 \mathrm{~mm} / \mathrm{mês}$.

Das sete vezes em que se procedeu à colheita de amostras de conjuntiva, também se verificou crescimento de fungos anemófilos no ar da sala. Em quatro casos encontraram-se as mesmas espécies de fungo no ar da sala e na conjuntiva (Tabela 5). Em nenhuma ocasião houve isolamento de fungos leveduriformes no ar da sala de colheita.

\section{DISCUSSÃO}

Indivíduos diabéticos são considerados mais predispostos às infecções do que as pessoas normais devido alterações na imunidade geradas pela glicemia elevada. A condição pri- mordial para ocorrer infecção micótica no olho, seria a presença de fungos na superfície ocular ${ }^{(39)}$. Neste estudo foi evidenciada existência de micobiota em conjuntiva sadia de indivíduos diabéticos, residentes na zona urbana, sendo identificado 11 gêneros diferentes de fungos nesses indivíduos. Nas maiores séries em que foi pesquisado fungo em conjuntiva sadia de indivíduos não diabéticos, o número de fungos isolados variou de sete a 20 gêneros ${ }^{(2,4,10-12,28)}$. Foi também observada, a presença de fungos anemófilos nas salas de exame, apesar dos cuidados tomados durante o processo de colheita, conforme descritos no método deste estudo. Dos fungos isolados do ar, apenas Exserohilum rostratum e Scytalidium hyalinum não foram relatados como pertencentes à micobiota anemófila normal da cidade de São Paulo ${ }^{(13,40)}$.

Dos gêneros de fungos isolados na conjuntiva dos diabé-

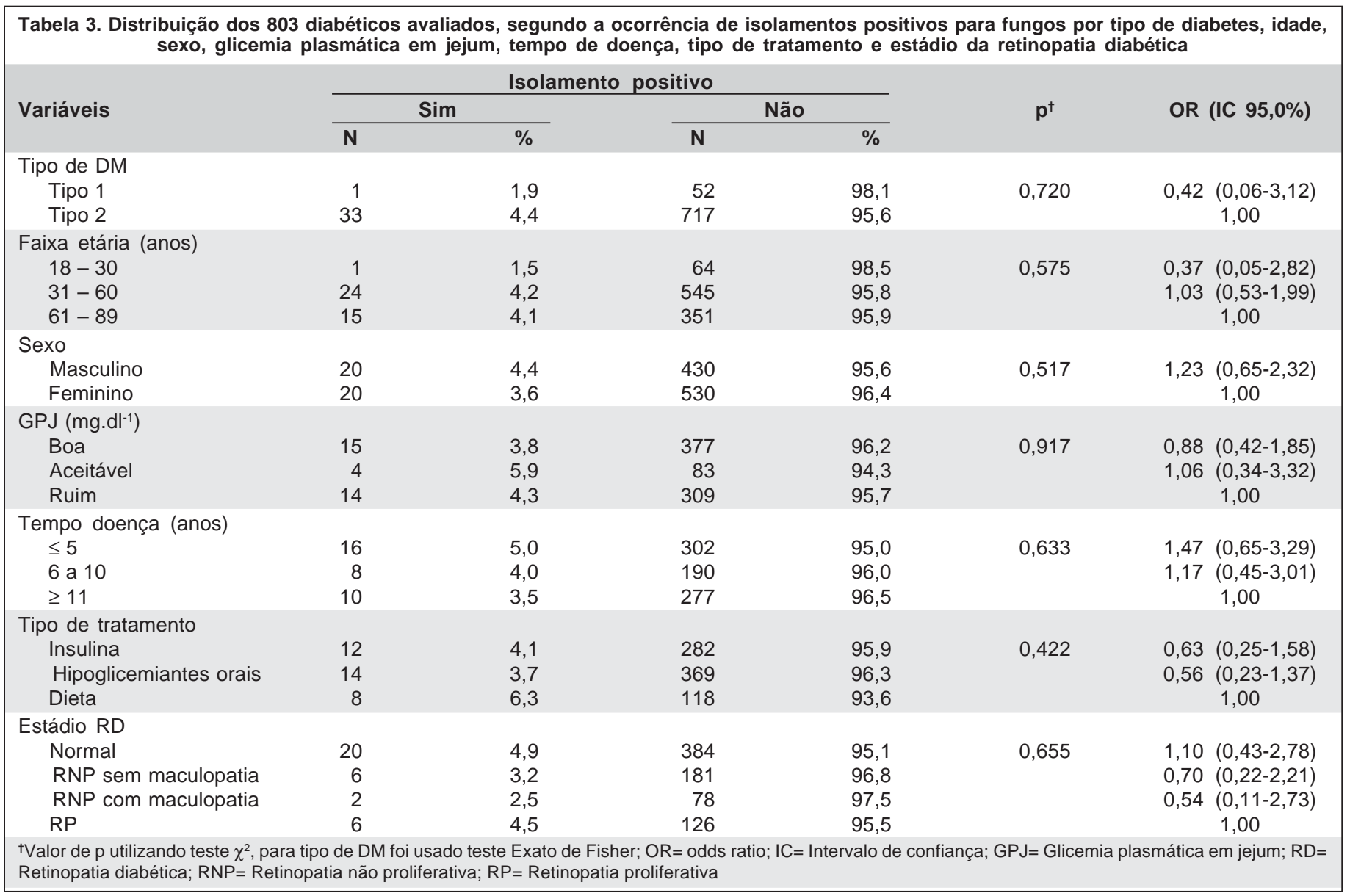

\begin{tabular}{|c|c|c|c|c|}
\hline $\begin{array}{l}\text { Valor da precipitação nos meses } \\
\text { de estudo (média em } \mathrm{mm} / \mathrm{mês} \text { ) }\end{array}$ & $\begin{array}{l}\text { Colheitas } \\
\text { N }\end{array}$ & \multicolumn{2}{|c|}{ Fungos isolados } & $p^{\dagger}$ \\
\hline Alta (250) & 456 & 41 & $9,0 \%$ & \\
\hline
\end{tabular}




\begin{tabular}{|c|c|c|}
\hline \multirow{2}{*}{$\begin{array}{l}\text { Data da } \\
\text { colheita }\end{array}$} & \multicolumn{2}{|c|}{ Espécies isoladas* } \\
\hline & Do ar & Conjuntiva \\
\hline $\begin{array}{l}\text { Setembro } \\
2001\end{array}$ & $\begin{array}{l}\text { Aspergillus niger } \\
\text { Curvularia spp. } \\
\text { Mycelia sterilia }\end{array}$ & Aspergillus fumigatus \\
\hline $\begin{array}{l}\text { Novembro } \\
2001\end{array}$ & $\begin{array}{l}\text { Acremonium spp. } \\
\text { Aspergillus fumigatus } \\
\text { Cladosporium } \\
\text { cladosporoides } \\
\text { Mycelia sterilia } \\
\text { Phoma spp. }\end{array}$ & $\begin{array}{l}\text { Acremonium spp. } \\
\text { Aspergillus fumigatus } \\
\text { Aspergillus niger } \\
\text { Cladosporium } \\
\text { cladosporoides } \\
\text { Colletotrichum sp. } \\
\text { Mycelia sterilia } \\
\text { Paecilomyces variotti } \\
\text { Penicillium spp. } \\
\text { Phoma spp. } \\
\text { Scytalidium lignicola } \\
\text { Ulocladium sp. }\end{array}$ \\
\hline $\begin{array}{l}\text { Fevereiro } \\
2002\end{array}$ & $\begin{array}{l}\text { Aspergillus niger } \\
\text { Penicillium citrinum }\end{array}$ & $\begin{array}{l}\text { Aspergillus spp. } \\
\text { Fonsecaea pedrosoi } \\
\text { Paecilomyces lilacinus } \\
\text { Penicillium citrinum }\end{array}$ \\
\hline $\begin{array}{l}\text { Março } \\
2002\end{array}$ & $\begin{array}{l}\text { Aspergillus niger } \\
\text { Exserohilum rostratum } \\
\text { Nattrassia mangiferae } \\
\text { Scytalidium hyalinum }\end{array}$ & $\begin{array}{l}\text { Aspergillus fumigatus } \\
\text { Aspergillus niger }\end{array}$ \\
\hline
\end{tabular}

ticos, verificou-se que oito dos 11 gêneros já haviam sido isolados nas placas expostas ao ar, havendo contudo, fungos presentes no ar e não isolados em conjuntiva e vice-versa. Das investigações que utilizaram placas com meios de cultivos expostos ao ar, por determinados intervalos de tempo, nos locais de colheita, os resultados variaram de nenhum isolamento a $2 \%$ dos cultivos positivos ${ }^{(2,6,10-12,14-15,24-25)}$. Em dois trabalhos, existe descrições de coincidências das espécies fúngicas isoladas do ar e da conjuntiva ${ }^{(6,12)}$.

Nos estudos com objetivos similares ao deste trabalho, observam-se algumas diferenças nos métodos que podem justificar os discrepantes resultados descritos. Essas diferenças consistem em melhor caracterizar: tipo de amostra utilizada, forma de interpretação dos resultados (segundo número de cultivos positivos por olhos ou indivíduos avaliados), critérios de inclusão (considerando tempo prévio de abstinência de uso de drogas tópicas ou presença de doenças sistêmicas), cuidados na colheita e na prevenção de contaminações, local de colheita (conjuntiva tarsal superior ou inferior) e instrumentos e meios de cultivos utilizados na obtenção das amostras de conjuntiva ${ }^{(2-6,8-12,14-15,18-26,28-32)}$.

Na seleção da amostra em estudo sobre micobiota de conjuntiva, na maioria dos trabalhos são utilizadas amostras de conveniência, exceto em dois, a seleção foi randomizada ${ }^{(2-6,8-12-14-15,18-26,28-32)}$.

Nesta investigação, os isolamentos de fungos na conjunti- va de indivíduos diabéticos não foram correlacionados, segundo a lateralidade. Este fato não sugere que haveria mais informação, se esta variável fosse avaliada, segundo o número de olhos com isolamentos de fungos. Pois, a identificação de fungos num olho, permite o diagnóstico da existência de micobiota em conjuntiva sadia nesse indivíduo ${ }^{(41)}$. A forma de interpretação, mais comumente utilizada na literatura para os isolamentos de fungos em conjuntiva sadia, é baseada no número de indivíduos com fungos nos olhos ${ }^{(2-4,6,8-11,14-15,18-19,22-25,28,30-32)}$. Na avaliação do grau da retinopatia diabética, também se observou que para a interpretação das avaliações das retinas dos diabéticos, caso a análise fosse realizada por indivíduo, forneceria informação similar, se analisada por olhos. Sendo utilizado para diagnóstico de retinopatia diabética, o estádio do indivíduo. Com isto, foi evitado aumento desnecessário do tamanho da amostra e maior precisão nos resultados.

Um dos principais critérios de inclusão, utilizado em estudos sobre micobiota em conjuntiva sadia, é a ausência de doenças oculares de superfície ou intra-ocular ${ }^{(2-6,8-11,14-15,19,24-26,29-31)}$. Em alguns trabalhos, é observado que os autores consideraram como portadores de conjuntivas normais, indivíduos com sinais clínicos de tracoma cicatricial ${ }^{(24)}$ e em uso de drogas antiglaucomatosas ${ }^{(7)}$ ou em tratamento de uveíte. Em outros estudos, os critérios de inclusão não foram descritos ${ }^{(4,18,22-23,25)}$.

A instilação de drogas na conjuntiva, independente do tipo do composto ativo presente, pode alterar a composição do filme lacrimal por efeito de lavagem da superfície ocular ${ }^{(42)}$. Muitos pesquisadores não consideraram o uso de colírio, como critério de inclusão ${ }^{(3-4,8,10)}$. Enquanto que outros, estipularam intervalos de tempo sem uso de medicamentos tópicos. Este prazo não foi mencionado em alguns estudos, em outros variou de 30 dias a seis meses e até um ano ${ }^{(2,5-6,9,11,14-15,19,24-26)}$.

A presença de indivíduos diabéticos entre as pessoas avaliadas quanto à presença de fungos em conjuntiva, ocorre em um trabalho, não havendo associação com isolamentos de fungos $^{(12)}$. A ocorrência de DM, como condição para exclusão das pessoas avaliadas, não foi descrita em nenhuma investigação $^{(2-6,8-11,14-15,18-26,28-32)}$.

$\mathrm{Na}$ realização do teste de esterilidade das zaragatoas, antes da colheita, procura-se comprovar a validade da esterilidade desses materiais. Na literatura, há relato somente de um pesquisador que utilizou este procedimento pré-colheita ${ }^{(3)}$. Outros autores avaliaram o tempo de exposição das zaragatoas estéreis ao ar, no local da colheita, não sendo considerado fator de contaminação no meio de cultivo ${ }^{(2,10)}$. O halo de calor gerado por chama de lamparina comum, colocada próximo do local de semeadura, foi usada para diminuir a taxa de contaminação por via aérea. Assemelhando-se a procedimentos realizados em outras investigações, utilizando bico de Bunsen $^{(14-15)}$.

No estudo da micobiota conjuntival, o tarso inferior tem sido o local mais freqüente para raspagem com zaragatoas ou espátula de platina ${ }^{(2,4,7-8,10,12,19,22-24,26)}$. Em algumas pesquisas, a colheita se realizou na conjuntiva tarsal superior ${ }^{(9)}$ e em 
outras, em ambos os sítios $^{(3,5)}$. Dos locais de colheita, não foram observadas diferenças significantes quanto à ocorrência de isolamentos.

Em estudos microbiológicos da conjuntiva humana, a forma mais utilizada para se obter material é o raspado, podendo ser realizado com zaragatoa seca ${ }^{(5,10,19,25-26,30-31)}$, zaragatoa umedecida em solução salina estéril ${ }^{(2,12,14-15,23,29,32)}$ ou umedecida em meio de cultivo ${ }^{(6-7,11)}$ e raspado com espátula de platina ${ }^{(3-4,8-9,918,22)}$.

A positividade dos cultivos encontrados com este instrumento para raspado conjuntival foi semelhante. Os resultados obtidos neste trabalho, com uso de zaragatoa umedecida em solução salina estéril, não diferiram dos demais.

Em estudo de citologia de saco conjuntival, quando comparadas às formas de colheita de conjuntiva, foi evidenciado que os resultados de raspado com uso de zaragatoas secas ou úmidas são superiores aos realizados com espátula de platina, pois nesta ocorre mais traumatismo da amostra. Além disto, com uso de zaragatoas secas se obtém maior número de células epiteliais ${ }^{(43)}$.

A taxa de crescimento dos fungos é altamente dependente da quantidade de amostra inoculada. Normalmente, amostras de conjuntiva apresentam quantidade reduzida de material, sendo prudente não usar meio de transporte e fazer a semeadura direta no meio de cultivo para primo-isolamento ${ }^{(44)}$. Alguns autores, em quatro estudos, utilizaram meios de transporte em amostras de conjuntiva. A positividade do cultivo para fungos, nos respectivos estudos, variou de 5,7 a $78,5 \%{ }^{(3,19,23,27)}$.

Neste experimento, utilizou-se o método da gravidade para obtenção das amostras de fungos do ar. Este método já foi aplicado em trabalhos com objetivos similares ${ }^{(13-15,40)}$. Observa-se mais comumente o uso de exposições entre 10 a 30 minutos $^{(12-15,40)}$. Alguns fatos sugerem que fungos anemófilos, presentes nas salas de colheita deste estudo, podem não estar associados ou influenciados nos resultados dos cultivos das amostras de conjuntiva, pois várias pessoas avaliadas eram procedentes de bairros diferentes dos locais de colheita. Essas pessoas ficaram em média, cinco minutos na sala de colheita, não havendo presença de fluxo de ar corrente no interior da sala. Tem sido citado que indivíduos expostos por longo prazo a ambientes fechados, exemplo: detentos, podem ter suas conjuntivas contaminadas pela micobiota anemófila do local ${ }^{(4,30)}$. Outros autores ampliam essa associação também a ambientes abertos ${ }^{(6-7,10,12)}$.

A micobiota da conjuntiva sadia, identificada nos indivíduos participantes deste estudo foi reduzida. O número de colônias de fungos presentes na conjuntiva dos indivíduos avaliados, variou de uma a 11 colônias por gênero. Em metade dos fungos isolados foi observado crescimento de apenas uma colônia. Semelhantes resultados foram achados em alguns trabalhos $^{(6,12)}$; em outros, foi verificado maior número de colônias por gênero isolado ${ }^{(14-15)}$. Analisando os resultados deste estudo, pelo baixo número de colônias em cada cultivo ${ }^{(6,12)}$ e alta freqüência de isolamentos monoculares ${ }^{(10)}$, é sugestivo que a micobiota isolada na conjuntiva dos diabéticos dessa amostra seja transitória, significando que, caso se repetisse os cultivos nos indivíduos avaliados, haveria pouca possibilidade de se encontrar fungos nas mesmas conjuntivas, ou caso houvesse isolamentos positivos nessas conjuntivas, a ocorrência das mesmas espécies, seria pouco provável.

Nesta pesquisa não foi repetido o cultivo dos casos positivos, porque tem sido desaconselhada esta abordagem em diversas investigações ${ }^{(3-4,6-7,10-11,16)}$.

Segundo dados climatológicos, obtidos continuamente próximos aos locais de colheita das amostras, não foram observadas diferenças nas médias da temperatura do ar e da precipitação de chuvas entre as regiões dos locais de colheita no período deste estudo. Os dados dessas estações são representativos das características climáticas da cidade de São Paulo $^{(38)}$.

Em alguns estudos, é avaliada a influência da sazonalidade sobre a micobiota conjuntival ${ }^{(11-12,17)}$. No presente estudo, em concordância com a literatura observamos maior número de isolamento de fungos em determinados meses com associação significante nos meses de maior taxa de precipitação de chuvas em relação àqueles com menores taxas ${ }^{(38)}$. Em trabalho realizado em zona rural da Índia, ocorreu associação entre baixa precipitação e maior número de fungos no $\mathrm{ar}^{(11)}$. Duas investigações realizadas na cidade de São Paulo sobre micobiota conjuntival em indivíduos portadores de síndrome da imunodeficiência adquirida, não revelaram variações significantes segundo a estação climática ou mês do ano.

Em relação aos diabéticos avaliados, as variáveis: idade, sexo, glicemia plasmática de jejum, tempo de diagnóstico, tipo de tratamento e grau de retinopatia não estão associadas à presença ou não de fungos na conjuntiva desses indivíduos.

As idades dos indivíduos diabéticos não foram homogêneas. Contudo, não foi observada influência da idade sobre a ocorrência de isolamentos positivos para fungos. Em apenas uma pesquisa foi verificada diferença significante segundo a idade $^{(15)}$.

Analisando a micobiota conjuntival segundo as faixas etárias dos indivíduos, verifica-se que esta variável sugere alguma interferência, de tal forma que, em estudos envolvendo recémnascidos, a positividade do cultivo de amostras de conjuntiva varia de $1,9 \%$ a $14,3 \%{ }^{(2,30)}$. Em crianças, a partir de dois meses de idade e jovens até 18 anos, os resultados variam de 1,5 a 43,5\% dos indivíduos ${ }^{(2,10,28)}$. Em pessoas com idades entre 19 e 85 anos, as frequiências obtidas variam de 3,2 a 72\% de indivíduos com isolamento fúngico positivo ${ }^{(2-3,10,14-15,25,28,30-32)}$.

Comparando-se a biota presente na conjuntiva, segundo a variável sexo, observa-se que as frequiências foram semelhantes em diversos estudos ${ }^{(2-6,8-12,14-15,18-26,28-32)}$. Na seleção dos diabéticos tipo 1 e tipo 2 não se observou distribuição homogênea segundo sexo, porém não houve influência desta variável na ocorrência, ou não, de isolamentos positivos nesses indivíduos. 
O tempo de diagnóstico de DM entre os indivíduos com DM tipo 1 foi maior do que os diabéticos tipo 2, porém, essa diferença não foi verificada em relação ao número de isolamentos em cada grupo. Nesta investigação, não houve controle do tipo de DM dos indivíduos, naturalmente pela história natural do DM tipo 1. É aceitável que esses pacientes tenham maior tempo de diagnóstico do que os diabéticos tipo $2^{(45)}$.

A manifestação de retinopatia diabética nos indivíduos analisados foi uniforme em relação ao tipo de DM, não sendo evidenciada associação com cultivos de fungos na conjuntiva. A presença de retinopatia diabética está relacionada com controle irregular da glicemia e com o tempo de diagnóstico ${ }^{(46)}$.

Em relação aos fungos isolados na conjuntiva dos indivíduos avaliados neste estudo, quase todos já haviam sido identificados em casos de infecções oculares, exceto os gêneros Ulocladium e Chaetomium ${ }^{(22,28,47-48)}$.

Concordando com este estudo, Aspergillus ssp. é o gênero mais isolado na maioria dos relatos sobre micobiota em conjuntiva sadia, em ceratite micótica e na micobiota anemófi$1 \mathrm{a}^{(2-6-8-12,14-15,18-26,28-30)}$. Em dois relatos, não foi identificado na conjuntiva $^{(31-32)}$.

O gênero Penicillium ssp. foi o segundo fungo mais freqüente da conjuntiva de diabéticos, confirmando resultados anteriores em trabalhos sobre micobiota de conjuntiva sadia de indivíduos normais ${ }^{(2-5,8,10-12,14-15,19-21,23-26,28-30)}$. Penicillium ssp. tem sido freqüentemente isolado em casos de ceratites micóticas e da biota do $\operatorname{ar}^{(16,40,48)}$.

Em relação aos gêneros Acremonium, Chaetomium, Colletotrichum, Fonsecaea, Penicillium, Phoma, Ulocladium e o fungo Mycelia sterilia, não foram encontradas descrições desses agentes causando infecções micóticas em diabéticos.

Fungos leveduriformes não foram isolados nesta pesquisa, porém são citados na maioria dos estudos sobre micobiota de conjuntiva sadia ${ }^{(2-6,8-12,14-15,18-25,28-32)}$.

A avaliação da micobiota conjuntival em indivíduos diabéticos, residentes em zona rural, seria um estudo interessante para elucidar dúvidas quanto à influência do ambiente, tomando-se cuidados semelhantes aos realizados neste estudo.

\section{CONCLUSÕES}

Observou-se que em diabéticos residentes em área urbana, com conjuntiva sadia, a biota ocular é composta de fungos. Não se observou associação significante entre número de isolamento fúngico e tipo de diabetes melito, idade, sexo, tempo de doença, tipo de tratamento e estádio da retinopatia diabética. O número de colônias de fungos por placas foi reduzido nessa população e acredita-se que apesar da micobiota existir na conjuntiva, seria necessária a ação de algum fator para permitir a geração de doença em olhos sadios. A despeito dos cuidados tomados na sala, durante o processo de colheita, verificou-se algumas coincidências entre as espécies fúngicas encontradas no ar e as identificadas na conjuntiva dos indivíduos estudados.

\section{AGRADECIMENTOS}

Aos alunos da Tecnologia Oftálmica da UNIFESP: Alessandro dos Santos Vidotti, Francine Tognin, Helga da Silva, Luana Mendieta, Maria Seiko, Sabrina de Souza Bonfim, Sheilla de Medeiros Correia e Vanessa Macedo Santa Cruz e a equipe do Laboratório de Microbiologia Ocular pelos diversos dias de trabalho que tivemos.

\section{ABSTRACT}

Purpose: To determine the mycobiota of the healthy conjunctiva in diabetic individuals, according to diabetes type, age, sex, disease time, type of treatment, and stage of diabetic retinopathy of the individuals. To identify the anemophilus mycobiota in the sampling rooms. Methods: A cross-sectional study was carried out on 803 diabetics who reside in the urban area of São Paulo-SP/Brazil. Sabouraud's dextrose agar culture with chloramphenicol was used for primoisolation, and the key of De Hoog was used to identify filamentous fungi. Results: Of the evaluated diabetics, 6.6\% (53/803) presented type 1 diabetes and $93.4 \%$ (750/803) type 2 . The positive cultures for fungi in the conjunctiva of diabetics was $4.2 \%$ (34/803), with $1.9 \%(1 / 53)$ in type 1 diabetics and $4.4 \%$ (33/740) in type 2 diabetics $(\mathrm{p}=0.720)$. With respect to the presence or not of isolated fungi, there was no statistically significant association regarding age $(\mathrm{p}=0.575)$, sex $(\mathrm{p}=0.517)$, disease time $(\mathrm{p}=0.633)$, type of treatment $(\mathrm{p}=0.422)$, and diabetic retinopathy stage $(\mathrm{p}=0.655)$ of the tested individuals. The identified fungi were all filamentous: Aspergillus spp. represented $59.5 \%(25 / 42)$ of isolations and $47.6 \%$ (20/42) of isolated species were Aspergillus niger. Growth of anemophilus fungi occurred in the air of the room and coincidences were observed between the isolated species from the air and those from the conjunctiva. Conclusions: Presence of mycobiota in healthy conjunctivas of diabetics was identified, with no significant association between the greater number of positive fungi isolations and the type of diabetes, age, sex, disease type, type of treatment, and stage of diabetic retinopathy. In the collection rooms, anemophilus mycobiota was identified.

Keywords: Conjunctiva/microbiology; Fungi/isolation \& purification; Diabetes mellitus; Diabetic retinopathy; Colony count, microbial, Aspergillus niger/isolation \& purification; Aspergillus/isolation \& purification; Urban population

\section{REFERÊNCIAS}

1. Cabral JH, Farah ALHL, Santos MAA, Belfort R, Mós EN, Farah ME. Microbiota anaeróbia e facultativa da conjuntiva normal de recém-nascidos. Arq Bras Oftalmol. 1993;56(3):138-45.

2. Hammeke JC, Ellis PP. Mycotic flora of the conjunctiva. Am J Ophthalmol. 1960;49:1174-8.

3. Ainley R, Smith B. Fungal flora of the conjunctival sac in healthy and diseased eyes. Br J Ophthalmol. 1965;49(10):505-15. 
4. Williamson J, Gordon AM, Wood R, Dyer AM, Yahya OA. Fungal flora of the conjunctival sac in health and disease. Influence of topical and systemic steroids. Br J Ophthalmol. 1968;52(2):127-37.

5. Sinha BN, Das MS. Bacterial and fungal flora of the conjunctival sacs in healthy and diseased eyes. J Indian Med Assoc. 1968;51(5):217-24.

6. Wilson LA, Ahearn DG, Jones DB, Sexton RR. Fungi from the normal outer eye. Am J Ophthalmol. 1969;67(1):52-6.

7. Olson CL. Fungal contamination of conjunctiva and lid margin. Arch Ophthalmol. 1969;81(3):351-5.

8. Cordero-Moreno R, Segovia-Diaz N. Flora micotica de la conjuntiva normal. Arch Soc Esp Oftalmol. 1973;33(9):829-36.

9. Costa ML, Galvão PG, Lage J. Flora micótica da conjuntiva de indivíduos normais. Rev Bras Oftalmol. 1975;34(2):199-206.

10. Gugnani HC, Gupta S, Talwar RS. Role of opportunistic fungi in ocular infections in Nigeria. Mycopathologia. 1977;65(1-3):155-66.

11. Sehgal SC, Dhawan S, Chhiber S, Sharma M, Talwar P. Frequency and significance of fungal isolations from conjunctival sac and their role in ocular infections. Mycopathologia. 1981;73(1):17-9.

12. Ando N, Takatori K. Fungal flora of the conjunctival sac. Am J Ophthalmol. 1982;94(1):67-74.

13. Gambale W, Purchio A, Paula CR. Influência de fatores abióticos na dispersão área de fungos na cidade de São Paulo, Brasil. Rev Microbial. 1983;14(3):204-14.

14. Scarpi MJ. Microbiota fúngica da conjuntiva normal de trabalhadores no corte de cana-de-açúcar. Rev Bras Oftalmol. 1985;44(1):57-65.

15. Vieira LA, Belfort Jr R, Fischman OF, Scarpi M. Estudo da flora fúngica da conjuntiva normal, da cana-de-açúcar e de anemófilos da região canavieira de Santa Rita - Paraíba (Brasil). Arq Bras Oftalmol. 1989;52(3):63-7.

16. Wilson LA, Ajello L. Agents of oculomycosis: fungal infections of the eye. In: Ajello L, Hay RJ, editors. Medical mycology. $9^{\text {th }}$ ed. London: Arnold; 1998. p.525-67.

17. Santos PM, Santos RCR, Muccioli C, Novo NF, Scarpi MJ, Gompertz OF. Variação sazonal da microbiota fúngica da conjuntiva de pacientes HIV soropositivos. Arq Bras Oftalmol. 1997;60(5):508-13.

18. Mitsui Y, Hanabusa J. Corneal infections after cortisone therapy. Br J Ophthalmol. 1955;39(4):244-50.

19. Agarwal LP, Khosla PK. Organismal conjunctival flora in Indians. Orient A Ophth 1963;1:145-52.

20. Fazakas A. Über die von Bindehaut, Hornhaut, Lidrand und Tränenwegen gezüchteten Pilze. Albrech Von Graefes Arch Ophthalmol. 1935;133:461-6.

21. Fazakas A. Über die Schimmelpilze des gesunden und kranken Auges. Graefes Arch Ophthalmol. 1938;138(4):416-23.

22. Azevedo ML. Investigações preliminares sobre a microflora ocular. Arq Bras Oftalmol. 1962;25(1):41-7.

23. Vasquez de Parga AS, Pereiro MM. Flora micotica de la conjuntiva. Arch Soc Oftal Hisp-Americana. 1965;25:168-81.

24. Nema HV, Ahuja OP, Mohapatra LN. Mycotic flora of the conjunctiva. Am J Ophthalmol. 1966;62(5):968-70.

25. Prabhaker H, Chitkara NL, Prabhaker BR. Mycotic and bacterial flora of the conjunctival sacs in healthy and diseased eyes. Indian J Pathol Bacteriol. 1969; 12(4):158-61.

26. Tomar VP, Sharma OP, Joshi K. Bacterial and fungal flora of normal conjunctiva. Ann Ophthalmol. 1971;3(6):669-71.

27. Raspiller A, Percebois G. [Micromycetes presence in the human conjunctival sac. Results from 1100 specimens and review of the literature]. Arch Ophtalmol Rev Gen Ophtalmol. 1972;32(4):311-22. French.
28. DeVoe AG, Silva-Hutner M. Fungal infections of the eye. In: Locatcher-Khorazo D, Seegal BC, editors. Microbiology of the eye. Saint Louis: C.V. Mosby; 1972. p.208-40.

29. Albesi EJ, Zapater R. Flora fungica de la conjuntiva en ojos sanos. Arch Oftalmol B Aires. 1972;47(11):329-31.

30. Segal E, Romano A, Eylan E, Stein R. Fungal flora of the normal conjunctival sac. Mykosen. 1977;20(1):9-14.

31. Miller B, Ellis PP. Conjunctival flora in patients receiving immunosuppressive drugs. Arch Ophthalmol. 1977;95(11):2012-4.

32. Cha SB, Fischman OF, Barros PSM, Mikoves R. Microbiota fúngica conjuntival de pacientes com síndrome da imunodeficiência adquirida (AIDS). Arq Bras Oftalmol. 1990;53(2):80-90.

33. The expert committee on the diagnosis and classification of diabetes mellitus. Report of the Expert Committee on the Diagnosis and Classification of Diabetes Mellitus. Diabetes Care. 1997;20(7):1183-97. Comment in: Diabetes Care. 2001;24(11):2017-8.

34. Warnock D. Introduction to the management of fungal infection in the compromised patient. In: Warnock D, Richardson M, editors. Fungal infection in the compromised patient. $2^{\text {nd }}$ ed. New York: John Wiley \& Sons; 1990. p.23-53.

35. Martins EN, Alvarenga LS, Höfling-Lima AL, Freitas D, Zorat-Yu MC, Farah $\mathrm{ME}$, et al. Aerobic bacterial conjunctival flora in diabetic patients. Cornea. 2004;23(2):136-42.

36. De Hoog GS, Guarro J, Gené J, Figueras MJ. Atlas of clinical fungi. $2^{\text {nd }}$ ed. Washington, DC: American Society for Microbiology; 2001.

37. Altman DG. Practical statistics for medical research. London: Chapman \& Hall; 1991.

38. Oliveira AP, Machado AJ. Variações regionais em São Paulo. In: Oliveira AP, Machado AJ, editors. Estudo observacional da radiação solar na cidade de São Paulo. São Paulo:Universidade de São Paulo, Instituto de Astronomia, Geofísica e Ciências Atmosféricas. [citado 2004 Abr 12]. Disponível em: http://www. iag.usp.br/meteo/labmicro/labmicro_02.htm

39. Richardson M, Shankland G. Pathogenesis of fungal infection in the non-compromised host. In: Warnock D, Richardson M, editors. Fungal infection in the compromised patient. $2^{\text {nd }}$ ed. Chichester: John Wiley \& Sons; 1991. p.1-22.

40. Gambale W, Purchio A, Paula CR. Periodicidade diária de fungos anemófilos na cidade de São Paulo, Brasil. Rev Microbiol. 1981;12(4):176-81.

41. Murdoch IE, Morris SS, Cousens SN. People and eyes: statistical approaches in ophthalmology. Br J Ophthalmol. 1998;82(8):971-3.

42. Abelson MB, Chambers WA, Smith LM. Conjunctival allergen challenge. A clinical approach to studying allergic conjunctivitis. Arch Ophthalmol 1990; 108(1):84-8.

43. Duszynski LR. Citology of the conjunctival sac: a quantitative and qualitative study. Am J Opthalmol. 1954;37(4):576-8.

44. Kwon-Chung KJ, Bennett JE. Laboratory diagnosis. In: Kwon-Chung KJ, Bennett JE, editors. Medical mycology. Philadelphia: Lea \& Febiger; 1992. p.44-71.

45. American Diabetes Association. Standards of medical care for patients with diabetes mellitus. Diabetes Care. 2002;25(Suppl 1):S33-49.

46. Klein R. The diabetes control and complications trial. In: Kertes PJ, Conway MD, editors. Clinical trials in ophthalmology: a summary and practice guide. Baltimore: Williams \& Wilkins; 1998. p.49-69.

47. Jones BR. Principles in the management of oculomycosis. XXXI Edward Jackson memorial lecture. Am J Ophthalmol. 1975;79(5):719-51.

48. Andrade AJM, Vieira LA, Hofling-Lima AL, Yu MCZ, Gompertz OF. Análise laboratorial de ceratites fúngicas em serviço universitário. Arq Bras Oftalmol. 2000;63(1):59-63. 